\title{
The Value Functions Approach and Hopf-Lax Formula for Multiobjective Costs via Set Optimization
}

\author{
Andreas Heinrich Hamel and Daniela Visetti \\ Free University of Bozen-Bolzano, Italy.
}

June 30, 2019

\begin{abstract}
The complete-lattice approach to optimization problems with a vector- or even set-valued objective already produced a variety of new concepts and results and was successfully applied in finance, statistics and game theory. For example, the duality issue for multi-criteria and vector optimization problems could be solved using the complete-lattice approach, compare [11]. So far, it has been applied to set-valued dynamic risk measures (in the stochastic case), as discussed in Feinstein, Rudloff etc. (see [11], for example), but it has not been applied to deterministic calculus of variations and optimal control problems.
\end{abstract}

In this paper, the following problem of set-valued optimization is considered: minimize the functional

$$
\bar{J}_{t}[y]=\int_{0}^{t} \bar{L}(s, y(s), \dot{y}(s)) d s+U_{0}(y(0))
$$

over all admissible $\operatorname{arcs} y$, where $\bar{L}$ is the associated multifunction to a vector-valued Lagrangian $L$, the integral is in the Aumann sense and $U_{0}$ is the initial cost. A new concept of value function, for which a Bellman's optimality principle holds, is introduced. Also the classical result of the Hopf-Lax formula holds for the generalized value function. Finally, a derivative with respect to the time $t$ and a directional derivative with respect to $x$ of the value function are defined, based on ideas close to the concepts in [12]. The value function is proved to be solution of a suitable Hamilton-Jacobi equation.

Keywords: multicriteria calculus of variations; value function; Hopf-Lax formula; Bellman's principle; Hamilton-Jacobi equation; set relations

Mathematics Subject Classification 49L20, 49L99, 90C29

\section{Introduction}

Despite the huge importance of multi-criteria decision making, the literature on calculus of variations and optimal control problems with multiple criteria is comparably poor. In particular, clear-cut multicriteria or even set-valued extensions of standard results like the Hamilton-Jacobi equation (and the value function approach in general) or Pontrjagin's maximum principle are still missing. Some early references are $[23,18]$ and due to Leitmann and $\mathrm{Yu}[22,21]$.

A more recent example is [4] in which the authors try to single out particular Pareto optimal solutions (=non-dominated objective values with respect to the componentwise order). This can be considered as a kind of a "second level" optimization. 
Applications include multicriteria caculus of variations problems in architecture [16] (compare [20] for a more general overview), and such problems lie within the framework of the present paper.

The major difficulty is the missing infimum (and supremum) in higher dimensions: if the order, e.g., in $\mathbb{R}^{d}$ is generated by a convex cone, it is not total in general and the infimum of a subset of $\mathbb{R}^{d}$ with respect to this order either does not exist or is not very useful since, in case of existence, it can be "far away from the set." Therefore, it is not a priori clear what the value function of a multi-criteria problem is and how to generalize a formula like

$$
\frac{\partial}{\partial t} V(x, t)+\min _{u}\{\nabla V(x, t) \cdot F(x, u)+C(x, u)\}=0
$$

to the case of several criteria.

The often chosen way out of this dilemma is scalarization: the most popular scalarization uses a weighted sum of the criteria, see [7] for a general overview and alternatives. In [6] and [10], a multicriteria optimal control problem is studied, where the preference relation is based respectively on the lexicographic order and on a pointed convex cone containing the origin. The main methodology also relies on scalarization. In [13], the authors consider the problem of optimally controlling a system of ordinary differential equations or of stochastic differential equations with respect to a vector-valued cost functional. In the deterministic case, for any direction in the dual cone they find a Pareto minimal vectorial cost, that is defined as the value function in the given direction. Its scalar product with the direction is a viscosity solution of a scalar Hamilton-Jacobi equation depending on the direction.

In this paper, we apply recent developments in set optimization to multi-criteria calculus of variations problems. This brings the infimum and supremum back into play. However, they are taken with respect to set relations, thus the original multi-criteria problem is first re-written as a set-valued one. This allows a generalized concept of value fuction, that gives results strikingly parallel to the scalar case. Attention is paid to formulate appropriate differentiability notions for the set-valued functions. Our findings include Bellman's optimality principle for a set-valued value function and formulas of Hopf-Lax type under convexity assumptions. Moreover, a parametrized familiy of Hamilton-Jacobi equations is obtained as multicriteria counterpart for the scalar HJ equation where the parametrization runs through the elements of the dual of the ordering cone.

It is worth noting that our set-valued value function cannot be reduced to the "point-plus-cone" case (i.e., a vector-valued function) in general, even if the original problem is vector-valued.

\section{Preliminaries}

The Minkowski sum of two non-empty sets $A, B \subseteq \mathbb{R}^{d}$ is $A+B=\{a+b \mid a \in A, b \in B\}$. It is extended to the whole power set $\mathcal{P}\left(\mathbb{R}^{d}\right)$ by

$$
\emptyset+A=A+\emptyset=\emptyset
$$

We also use $A \oplus B:=\operatorname{cl}(A+B)$, the "closed sum" of two sets.

A set $C \subseteq \mathbb{R}^{d}$ is a cone if $s C \subseteq C$ for all $s>0$, and it is a convex cone if additionally $C+C \subseteq C$. Thus, 0 does not necessarily belong to a convex cone. The (positive) dual of a cone $C$ is defined as

$$
C^{+}=\left\{\zeta \in \mathbb{R}^{d} \mid \zeta \cdot z \geq 0\right\}
$$

We consider the following subsets of the power set $\mathcal{P}\left(\mathbb{R}^{d}\right)$ (see for instance [11]):

$$
\begin{aligned}
& \mathcal{P}\left(\mathbb{R}^{d}, C\right)=\left\{A \in \mathcal{P}\left(\mathbb{R}^{d}\right) \mid A=A+C\right\} \\
& \mathcal{F}\left(\mathbb{R}^{d}, C\right)=\left\{A \in \mathcal{P}\left(\mathbb{R}^{d}\right) \mid A=A \oplus C\right\} \\
& \mathcal{G}\left(\mathbb{R}^{d}, C\right)=\left\{A \in \mathcal{P}\left(\mathbb{R}^{d}\right) \mid A=\operatorname{cl} \operatorname{co}(A+C)\right\}
\end{aligned}
$$


where $\mathrm{cl}$ and co are the closure and the convex hull, respectively.

The pairs $\left(\mathcal{P}\left(\mathbb{R}^{d}, C\right), \supseteq\right),\left(\mathcal{F}\left(\mathbb{R}^{d}, C\right), \supseteq\right),\left(\mathcal{G}\left(\mathbb{R}^{d}, C\right), \supseteq\right)$ are complete lattices. If $\mathcal{A} \subseteq \mathcal{P}\left(\mathbb{R}^{d}, C\right)$, then the infimum and the supremum of $\mathcal{A}$ are given by

$$
\inf \mathcal{A}=\bigcup_{A \in \mathcal{A}} A \quad \sup \mathcal{A}=\bigcap_{A \in \mathcal{A}} A .
$$

While the supremum in $\left(\mathcal{F}\left(\mathbb{R}^{d}, C\right), \supseteq\right),\left(\mathcal{G}\left(\mathbb{R}^{d}, C\right), \supseteq\right)$ is given by the same intersection formula as in $\left(\mathcal{P}\left(\mathbb{R}^{d}, C\right), \supseteq\right)$, one has

$$
\inf \mathcal{A}=\operatorname{cl} \bigcup_{A \in \mathcal{A}} A \quad \text { and } \quad \inf \mathcal{A}=\operatorname{clco} \bigcup_{A \in \mathcal{A}} A,
$$

for $\mathcal{A} \subseteq \mathcal{F}\left(\mathbb{R}^{d}, C\right)$ and $\mathcal{A} \subseteq \mathcal{G}\left(\mathbb{R}^{d}, C\right)$, respectively. The order relation $\supseteq$ is the same in all three sets (of subsets of $\mathbb{R}^{d}$ ). Let $\mathcal{A}$ be a subset of one of the three. An element $A_{0} \in \mathcal{A}$ is called minimal for $\mathcal{A}$ if

$$
A \in \mathcal{A}, A \supseteq A_{0} \Longrightarrow A=A_{0} .
$$

The set of all minimal elements of $\mathcal{A}$ is denoted by $\operatorname{Min} \mathcal{A}$, and it will be clear from the context if it is meant in $\mathcal{P}\left(\mathbb{R}^{d}, C\right), \mathcal{F}\left(\mathbb{R}^{d}, C\right)$ or $\mathcal{G}\left(\mathbb{R}^{d}, C\right)$.

A set $\mathcal{A}$ is said to satisfy the domination property if for any $A \in \mathcal{A}$, there exists $A_{0} \in \operatorname{Min} \mathcal{A}$ such that $A_{0} \supseteq A$.

Let $\zeta \in C^{+} \backslash\{0\}$ and let

$$
H^{+}(\zeta)=\left\{z \in \mathbb{R}^{d} \mid \zeta \cdot z \geq 0\right\}
$$

where $\zeta \cdot z$ denotes the usual scalar product. For two sets $A, B \in \mathcal{P}\left(\mathbb{R}^{d}, C\right)$, the set

$$
A-{ }_{\zeta} B=\left\{z \in \mathbb{R}^{d} \mid z+B \subseteq A \oplus H^{+}(\zeta)\right\}
$$

is called the $\zeta$-difference of $A$ and $B$.

Remark 2.1. For any set $A \subseteq \mathbb{R}^{d}, A \oplus H^{+}(\zeta)$ is either $\emptyset, \mathbb{R}^{d}$ or a closed (shifted) half-space. Therefore, one has

$$
A-{ }_{\zeta} B=\left\{z \in Z \mid \zeta \cdot z+\inf _{b \in B} \zeta \cdot b \geq \inf _{a \in A} \zeta \cdot a\right\}
$$

where it is understood that $\inf _{y \in \emptyset} \zeta \cdot y=+\infty$ and $r+(-\infty)=-\infty$ as well as $r+(+\infty)=+\infty$ for $r \in \mathbb{R}$. See [12,11] for more details on this set difference which is a version of the so-called geometric or Pontryagin difference.

The following is a technical lemma that will be used in a forthcoming section.

Lemma 2.2. Let $A, B, D \in \mathcal{P}(Z, C)$ and $\zeta \in C^{+} \backslash\{0\}$.

(i) Assume $A \neq \emptyset$ and either $B \oplus H^{+}(\zeta)=Z$ or $B \oplus H^{+}(\zeta) \neq Z, D \oplus H^{+}(\zeta) \neq Z$. Then

$$
A+B \supseteq D \quad \Longrightarrow \quad A+\left(B-{ }_{\zeta} D\right) \supseteq H^{+}(\zeta) .
$$

(ii) Assume $A=\emptyset$, or $A, D \neq \emptyset$ and $B \oplus H^{+}(\zeta) \neq Z$. Then,

$$
A+B \subseteq D \oplus H^{+}(\zeta) \quad \Longrightarrow \quad A \oplus\left(B-{ }_{\zeta} D\right) \subseteq H^{+}(\zeta)
$$

Proof. (i) If $B \oplus H^{+}(\zeta)=Z$, then $B-{ }_{\zeta} D=Z$, hence $A \oplus\left(B-{ }_{\zeta} D\right)=Z$, and the claim is trivially satisfied. Let $B \oplus H^{+}(\zeta) \neq Z$. If $B=\emptyset$, then the assumption implies $D=\emptyset$, and again the claim is trivial. Finally, assume $B \oplus H^{+}(\zeta) \notin\{Z, \emptyset\}$. Then, there is $z_{B} \in Z$ with $z_{B}+H^{+}(\zeta)=B \oplus H^{+}(\zeta)$, 
and by assumption there also is $z_{D} \in Z$ with $z_{D}+H^{+}(\zeta)=D \oplus H^{+}(\zeta)$. In this case, one has $B-{ }_{\zeta} D=z_{B}-z_{D}+H^{+}(\zeta)$ and hence

$$
\inf _{z \in A+\left(B-{ }_{\zeta} D\right)} \zeta \cdot z=\inf _{a \in A} \zeta \cdot a+\zeta \cdot z_{B}-\zeta \cdot z_{D} .
$$

On the other hand, the assumption implies

$$
\zeta \cdot z_{D} \geq \inf _{a \in A} \zeta \cdot a+\zeta \cdot z_{B}
$$

The last two formulas together imply $\inf _{z \in A+(B-\zeta D)} \zeta \cdot z \leq 0$ which proves the claim.

(ii) The implication is trivial for $A=\emptyset$. If $A, D \neq \emptyset$ and $B \oplus H^{+}(\zeta) \neq Z$, then there are two cases. First, if $D \oplus H^{+}(\zeta)=Z$, then $B-{ }_{\zeta} D=\emptyset$ and the implication is trivial. Secondly, if $D \oplus H^{+}(\zeta)=z_{D}+H^{+}(\zeta)$ for some $z_{D} \in Z$, then the assumption implies either $B=\emptyset$ (the implication is trivial again) or $B \oplus H^{+}(\zeta)=z_{B}+H^{+}(\zeta)$ for some $z_{B} \in Z$. In the latter case, the assumption implies $A \oplus H^{+}(\zeta)=z_{A}+H^{+}(\zeta)$ for some $z_{A} \in Z$, and one can get the conclusion by similar arguments as for (i).

Let $\left\{A_{n}\right\}_{n \in \mathbb{N}}$ be a sequence of sets in $\mathcal{P}\left(\mathbb{R}^{d}, C\right)$, we denote by $\lim _{n \rightarrow \infty} A_{n}$ the following set:

$$
\lim _{n \rightarrow \infty} A_{n}=\left\{z \in \mathbb{R}^{d} \mid \forall n \in \mathbb{N}, \exists z_{n} \in A_{n}, \lim _{n \rightarrow \infty} z_{n}=z\right\} .
$$

This definition of limit coincides with the upper limit of Painlevé-Kuratowski $\left(\operatorname{Liminf}_{n \rightarrow \infty} A_{n}=\right.$ $\left\{z \in Z \mid \lim _{n \rightarrow \infty} d\left(z, A_{n}\right)=0\right\}$, see [1]).

Let $\left\{A_{s}\right\}_{s \in S}$ with $S \subseteq \mathbb{R}$ be a family of sets in $\mathcal{P}\left(\mathbb{R}^{d}, C\right)$ and $\bar{s} \in \mathbb{R}$. We denote by $\lim _{s \rightarrow \bar{s}} A_{s}$ the set which satisfies that for any sequence $\left\{s_{n}\right\}_{n \in \mathbb{N}} \subseteq S$ with $s_{n} \rightarrow \bar{s}$ one has

$$
\lim _{s \rightarrow \bar{s}} A_{s}=\lim _{n \rightarrow \infty} A_{s_{n}} .
$$

Let $f$ be a function $f: \mathbb{R}^{n} \rightarrow \mathcal{P}\left(\mathbb{R}^{d}, C\right)$. The graph of $f$ is

$$
\text { graph } f=\left\{(x, z) \in \mathbb{R}^{n} \times \mathbb{R}^{d} \mid z \in f(x)\right\} .
$$

The domain of $f$ is

$$
\operatorname{dom} f=\left\{x \in \mathbb{R}^{n} \mid f(x) \neq \emptyset\right\}
$$

The function is convex if and only if graph $f$ is a convex subset of $\mathbb{R}^{n} \times \mathbb{R}^{d}$. This is equivalent to the following condition: for any $\lambda \in(0,1), x_{1}, x_{2} \in \mathbb{R}^{n}$

$$
f\left(\lambda x_{1}+(1-\lambda) x_{2}\right) \supseteq \lambda f\left(x_{1}\right)+(1-\lambda) f\left(x_{2}\right) .
$$

Let $X$ be a separated (Hausdorff) topological space, $Z$ be a separated (Hausdorff) topological vector space and $\Gamma: X \rightarrow \mathcal{P}(Z)$ a set-valued function. The domain of $\Gamma$ is:

$$
\operatorname{dom} \Gamma=\{x \in X \mid \Gamma(x) \neq \emptyset\} .
$$

We recall the following continuity definitions for set-valued functions (see [9]).

Let $\mathcal{N}_{Z}$ denote the class of balanced neighborhoods of $0 \in Z$. If $x_{0} \in X$ and $\mathcal{N}_{X}\left(x_{0}\right)$ is the set of neighborhoods of $x_{0}$,

(a) $\Gamma$ is Hausdorff upper continuous at $x_{0}$ if for all $B \in \mathcal{N}_{Z}$, there exists $A \in \mathcal{N}_{X}\left(x_{0}\right)$, such that for any $x \in A$

$$
\Gamma(x) \subset \Gamma\left(x_{0}\right)+B
$$


(b) $\Gamma$ is Hausdorff lower continuous at $x_{0}$ if for all $B \in \mathcal{N}_{Z}$, there exists $A \in \mathcal{N}_{X}\left(x_{0}\right)$, such that for any $x \in A$

$$
\Gamma\left(x_{0}\right) \subset \Gamma(x)+B
$$

(c) $\Gamma$ is Hausdorff continuous at $x_{0}$ if $\Gamma$ is Hausdorff upper continuous and Hausdorff lower continuous at $x_{0}$.

For extended vector-valued functions $F: X \rightarrow Z \cup\{+\infty\}$ and for $C \subset Z$ convex cone, the following continuity concepts can be considered.

If $x_{0} \in \operatorname{dom} F$, then

(i) $F$ is $C$-lower continuous at $x_{0}$ if for any $B \in \mathcal{N}_{Z}$, there exists $A \in \mathcal{N}_{X}\left(x_{0}\right)$, such that for any $x \in A$

$$
F(x) \in F\left(x_{0}\right)+B+(C \cup\{+\infty\})
$$

(ii) $F$ is $C$-upper continuous at $x_{0}$ if for all $B \in \mathcal{N}_{Z}$, there exists $A \in \mathcal{N}_{X}\left(x_{0}\right)$, such that for any $x \in A$

$$
F(x) \in F\left(x_{0}\right)+B-C
$$

Associated to the extended vector-valued function $F$ there is the set-valued function $\bar{F}: X \rightarrow$ $\mathcal{P}(Z \cup\{+\infty\})$, defined by $\bar{F}(x)=F(x)+C$ if $x \in \operatorname{dom} F$ and $\bar{F}(x)=\{+\infty\}$ if $x \notin \operatorname{dom} F$.

It is possible to prove (see [9]) that $F$ is $C$-lower continuous at $x_{0}$ if and only if $\bar{F}$ is Hausdorff upper continuous at $x_{0}$ and that $F$ is $C$-upper continuous at $x_{0}$ if and only if $\bar{F}$ is Hausdorff lower continuous at $x_{0}$.

Let $(X, \mathcal{A}, \mu)$ be a measure space and let $f$ be a set-valued map from $X$ into the closed nonempty subsets of $\mathbb{R}^{d}$.

The set of the integrable selections of $f$ is:

$$
\mathcal{F}=\left\{\varphi \in L^{1}\left(X, \mathbb{R}^{d}\right) \mid \varphi(x) \in f(x) \text { a.e. in } X\right\}
$$

The integral of $f$ on $\mathbb{R}^{n}$ is the set of integrals of the integrable selections of $f$ :

$$
\int_{X} f d \mu=\left\{\int_{\mathbb{R}^{n}} \varphi d \mu \mid \varphi \in \mathcal{F}\right\}
$$

In [15] the following Jensen inequality for set-valued functions has been proved:

Let $X, Z$ be Banach spaces and let $D \subseteq X$ be open and convex. If $\Gamma: D \subseteq \operatorname{dom} \Gamma \rightarrow \mathcal{F}(Z, C)$ is convex and Hausdorff continuous, then for each normalized measure space and for all $\mu$-integrable functions $\varphi: \Omega \rightarrow D$ such that $\operatorname{cl} \operatorname{co} \varphi(\Omega) \subseteq D$ we have

$$
\int_{\Omega}(\Gamma \circ \varphi) d \mu \subseteq \Gamma\left(\int_{\Omega} \varphi d \mu\right) .
$$

The integrals are meant here in the Aumann sense. We also observe that, being $\Gamma$ convex, more precisely $\Gamma: D \subseteq \operatorname{dom} \Gamma \rightarrow \mathcal{G}(Z, C)$.

Let $X$ be a non-empty set, $f: X \rightarrow \mathcal{F}(Z, C)$ a function and $f[X]=\{f(x) \mid x \in X\}$.

(a) A set $M \subset X$ is called an infimizer for $f$ if

$$
\inf f[M]=\inf f[X]
$$

(b) An element $x_{0} \in X$ is called a minimizer for $f$ if $f\left(x_{0}\right)$ is minimal for $f[X]$. 
(c) A set $M \subset X$ is called a solution of the problem minimize $f(x)$ subject to $x \in X$ if $M$ is an infimizer for $f$ and each $x_{0} \in M$ is a minimizer for $f$. It is called a full solution if the set $f[M]$ includes all minimal elements of $f[X]$.

Let $\eta \in \mathbb{R}^{n}$ and $\zeta \in C^{+}$be given. We recall the definition of the function $S_{(\eta, \zeta)}: \mathbb{R}^{n} \rightarrow \mathcal{G}\left(\mathbb{R}^{d}, C\right)$ :

$$
S_{(\eta, \zeta)}(x)=\left\{z \in \mathbb{R}^{d} \mid \zeta \cdot z \geq \eta \cdot x\right\} .
$$

Such a function is additive and positively homogeneous, i.e., for all $x \in \mathbb{R}^{n}, \lambda>0$

$$
S_{(\eta, \zeta)}(\lambda x)=\lambda S_{(\eta, \zeta)}(x)
$$

and for all $x_{1}, x_{2} \in \mathbb{R}^{n}$

$$
S_{(\eta, \zeta)}\left(x_{1}+x_{2}\right)=S_{(\eta, \zeta)}\left(x_{1}\right)+S_{(\eta, \zeta)}\left(x_{2}\right) .
$$

Let $\hat{z} \in \mathbb{R}^{d}$ be such that $\zeta \cdot \hat{z}=1$. Then for any $x \in \mathbb{R}^{n}$

$$
S_{(\eta, \zeta)}(x)=(\eta \cdot x) \hat{z}+H^{+}(\zeta)
$$

(see [11]).

The Fenchel conjugate of the function $f: \mathbb{R}^{n} \rightarrow \mathcal{P}\left(\mathbb{R}^{d}, C\right)$ is defined as the function

$$
\begin{aligned}
& f^{*}: \quad \mathbb{R}^{n} \times C^{+} \backslash\{0\} \quad \rightarrow \quad \mathcal{G}\left(\mathbb{R}^{d}, C\right) \\
& (\eta, \zeta) \quad \mapsto \sup _{x \in \mathbb{R}^{n}} S_{(\eta, \zeta)}(x)-z^{*} f(x)
\end{aligned}
$$

\section{Value function and Bellman's optimality principle}

For an introduction on the classical results, see for example [8], [14], [19] and [5].

Let us consider

$$
0<T<+\infty, \quad Q_{T}=[0, T] \times \mathbb{R}^{n}, \quad(t, x) \in \mathbb{R} \times \mathbb{R}^{n}
$$

and the continuous lower bounded functions

$$
L: Q_{T} \times \mathbb{R}^{n} \rightarrow \mathbb{R}^{d}, \quad U_{0}: \mathbb{R}^{n} \rightarrow \mathbb{R}^{d}
$$

where $L$ is the running cost or Lagrangian and $U_{0}$ is the initial cost.

We consider also $T=+\infty$, but in this case $Q_{\infty}$ is defined as $[0,+\infty) \times \mathbb{R}^{n}$.

For any $(t, x) \in Q_{T}$ (respectively $Q_{\infty}$ ), define the set of admissible arcs:

$$
Y(t, x)=\left\{y \in W^{1,1}\left([0, t], \mathbb{R}^{n}\right) \mid y(t)=x\right\} .
$$

We consider the problem of "minimizing" the cost functional $J_{t}: W^{1,1}\left([0, t], \mathbb{R}^{n}\right) \rightarrow \mathbb{R}^{d}$

$$
J_{t}[y]=\int_{0}^{t} L(s, y(s), \dot{y}(s)) d s+U_{0}(y(0))
$$

with respect to $y \in Y(t, x)$.

In order to formalize the definition of infimum in the lattice sense, we consider the functions:

$$
\begin{array}{rll}
\bar{L} & : & Q_{T} \times \mathbb{R}^{n} \rightarrow \mathcal{G}\left(\mathbb{R}^{d}, C\right) \\
\bar{J}_{t} & : & W^{1,1}\left([0, t], \mathbb{R}^{n}\right) \rightarrow \mathcal{G}\left(\mathbb{R}^{d}, C\right)
\end{array}
$$

defined by $\bar{L}(s, y, z)=L(s, y, z)+C$ and the integral in

$$
\bar{J}_{t}[y]=\int_{0}^{t} \bar{L}(s, y(s), \dot{y}(s)) d s+U_{0}(y(0))
$$

is in the Aumann sense (see [2] or [1]). 
Remark 3.1. The set $\bar{J}_{t}[y]$ coincides with $J_{t}[y]+C$ (here the integral is considered component-wise). In fact, for any $c \in C, L(s, y(s), \dot{y}(s))+c / t$ is an integrable selection of $\bar{L}(s, y(s), \dot{y}(s))$ and $J_{t}[y]+C \subseteq$ $\bar{J}_{t}[y]$. Vice versa, if $\eta \in \bar{J}_{t}[y]$, then $\eta=\int_{0}^{t} f(s) d s+U_{0}(y(0))$ where $f$ is an integrable selection of $\bar{L}(s, y(s), \dot{y}(s))$. This means that $f(s)=L(s, y(s), \dot{y}(s))+c(s)$ for a suitable $c(s) \in L^{1}([0, t], C)$ and $\eta \in J_{t}[y]+C$.

Our problem is then:

$$
\text { minimize } \bar{J}_{t}[y] \text { over all arcs } y \in Y(t, x) \text {. }
$$

Since the functional $\bar{J}_{t}$ maps into the complete lattices $\mathcal{G}\left(\mathbb{R}^{d}, C\right) \subset \mathcal{F}\left(\mathbb{R}^{d}, C\right)$, the infimum is now well defined.

In the classical real-valued theory, the value function was introduced as the infimum of the functional over all the admissible arcs. Here we give the following definition.

Definition 3.2. The functions

(i) $U: Q_{T} \rightarrow \mathcal{F}\left(\mathbb{R}^{d}, C\right)$

$$
U(t, x)=\inf _{y \in Y(t, x)} \bar{J}_{t}[y]=\mathrm{cl} \bigcup_{y \in Y(t, x)} \bar{J}_{t}[y]
$$

(ii) $U: Q_{T} \rightarrow \mathcal{G}\left(\mathbb{R}^{d}, C\right)$

$$
U(t, x)=\inf _{y \in Y(t, x)} \bar{J}_{t}[y]=\operatorname{clco} \bigcup_{y \in Y(t, x)} \bar{J}_{t}[y]
$$

are called value function of problem (2) with values in $\mathcal{F}\left(\mathbb{R}^{d}, C\right), \mathcal{G}\left(\mathbb{R}^{d}, C\right)$, respectively.

Remark 3.3. If $\bar{J}_{t}$ is convex (for example if $\bar{L}$ is convex), then

$$
\operatorname{cl} \bigcup_{y \in Y(t, x)} \bar{J}_{t}[y]=\operatorname{clco} \bigcup_{y \in Y(t, x)} \bar{J}_{t}[y]
$$

and the $\mathcal{F}\left(\mathbb{R}^{d}, C\right)$-valued $U$ is $\mathcal{G}\left(\mathbb{R}^{d}, C\right)$-valued.

In this section we consider the value function $U$ with values in $\mathcal{F}\left(\mathbb{R}^{d}, C\right)$.

Theorem 3.4 (Bellman's optimality principle). Let $(t, x) \in Q_{T}$ and $y \in Y(t, x)$. Then, for all $t^{\prime} \in[0, t]$,

$$
U(t, x) \supseteq \int_{t^{\prime}}^{t} \bar{L}(s, y(s), \dot{y}(s)) d s \oplus U\left(t^{\prime}, y\left(t^{\prime}\right)\right) .
$$

Moreover, the set $M \subseteq Y(t, x)$ is an infimizer for problem (2) if and only if for all $t^{\prime} \in[0, t]$,

$$
U(t, x)=\inf _{y \in M}\left(\int_{t^{\prime}}^{t} \bar{L}(s, y(s), \dot{y}(s)) d s \oplus U\left(t^{\prime}, y\left(t^{\prime}\right)\right)\right) .
$$

Proof. Given $t^{\prime} \in[0, t]$ and $y \in Y(t, x)$, we consider

$$
\xi(s)= \begin{cases}\eta(s), & \text { if } s \in\left[0, t^{\prime}\right] \\ y(s), & \text { if } s \in\left[t^{\prime}, t\right]\end{cases}
$$

where $\eta$ is any arc in $W^{1,1}\left(\left[0, t^{\prime}\right] ; \mathbb{R}^{n}\right)$ such that $\eta\left(t^{\prime}\right)=y\left(t^{\prime}\right)$. Then $\xi \in Y(t, x)$ and

$$
U(t, x) \supseteq \bar{J}_{t}[\xi]=\int_{t^{\prime}}^{t} \bar{L}(s, y(s), \dot{y}(s)) d s \oplus \int_{0}^{t^{\prime}} \bar{L}(s, \eta(s), \dot{\eta}(s)) d s+U_{0}(\eta(0)) .
$$


Taking the infimum over all $\eta \in Y\left(t^{\prime}, y\left(t^{\prime}\right)\right)$, we obtain (5).

If (6) holds for any $t^{\prime} \in[0, t]$, putting $t^{\prime}=0$ gives that $M$ is an infimizer. Vice versa, if $M$ is an infimizer $U(t, x)=\inf _{y \in M} \bar{J}_{t}[y]$. By (5), we have

$$
U(t, x) \supseteq \inf _{y \in M}\left(\int_{t^{\prime}}^{t} \bar{L}(s, y(s), \dot{y}(s)) d s+U\left(t^{\prime}, y\left(t^{\prime}\right)\right)\right) .
$$

Let us now suppose that $u \in U(t, x)$. Then either $u \in \bar{J}_{t}[y]$ for some $y \in M$ or it is in the closure of $\bigcup_{y \in M} \bar{J}_{t}[y]$. In the first case $u$ is in

$$
\begin{aligned}
& \int_{t^{\prime}}^{t} \bar{L}(s, y(s), \dot{y}(s)) d s \oplus \int_{0}^{t^{\prime}} \bar{L}(s, y(s), \dot{y}(s)) d s+U_{0}(y(0)) \\
& \quad \subseteq \int_{t^{\prime}}^{t} \bar{L}(s, y(s), \dot{y}(s)) d s \oplus U\left(t^{\prime}, y\left(t^{\prime}\right)\right) .
\end{aligned}
$$

In the other case $u$ will be in the closure of

$$
\bigcup_{y \in M}\left(\int_{t^{\prime}}^{t} \bar{L}(s, y(s), \dot{y}(s)) d s \oplus U\left(t^{\prime}, y\left(t^{\prime}\right)\right)\right),
$$

so in $\inf _{y \in M}\left(\int_{t^{\prime}}^{t} \bar{L}(s, y(s), \dot{y}(s)) d s+U\left(t^{\prime}, y\left(t^{\prime}\right)\right)\right)$.

\section{Hopf-Lax formula}

We consider now a Lagrangian function depending only on the last component $L(t, x, q)=L(q)$ and $T=+\infty$. The following classical theorem allows to pass from an infimum over the set of admissible $\operatorname{arcs} Y(t, x)$ to an infimum over $\mathbb{R}^{n}$.

Theorem 4.1. Let $L: \mathbb{R}^{n} \rightarrow \mathbb{R}^{d}$ and $U_{0}: \mathbb{R}^{n} \rightarrow \mathbb{R}^{d}$ be continuous functions and $\bar{L}: \mathbb{R}^{n} \rightarrow \mathcal{G}\left(\mathbb{R}^{d}, C\right)$ be a convex function. Then the value function $U$ with values in $\mathcal{G}\left(\mathbb{R}^{d}, C\right)$ is given by the formula:

$$
U(t, x)=\inf _{w \in \mathbb{R}^{n}}\left[t \bar{L}\left(\frac{x-w}{t}\right)+U_{0}(w)\right]
$$

for all $t>0$ and $x \in \mathbb{R}^{n}$. Let $F_{x, t}: \mathbb{R}^{n} \rightarrow \mathbb{R}^{d}$ and $\bar{F}_{x, t}: \mathbb{R}^{n} \rightarrow \mathcal{G}\left(\mathbb{R}^{d}, C\right)$ be defined by

$$
F_{x, t}(w)=t L\left(\frac{x-w}{t}\right)+U_{0}(w) \quad \text { and } \quad \bar{F}_{x, t}(w)=F_{x, t}(w) \oplus C,
$$

respectively. If the image $\bar{F}_{x, t}\left[\mathbb{R}^{n}\right]$ satisfies the domination property, there is at least one point $w_{0} \in \mathbb{R}^{n}$ that is a minimizer of $\bar{F}_{x, t}$ and a minimal point of $U(x, t)$.

Proof. Let us denote by $V(t, x)$ the right hand side of $(7)$. Let $(t, x) \in Q_{T}, w \in \mathbb{R}^{n}$ be fixed. Consider

$$
\xi(s)=w+\frac{s}{t}(x-w), \quad 0 \leq s \leq t .
$$

Then, $\xi \in Y(t, x)$ and consequently

$$
U(t, x) \supseteq \bar{J}_{t}[\xi]=t \bar{L}\left(\frac{x-w}{t}\right)+U_{0}(w) .
$$


Since this is true for all $w$

$$
U(t, x) \supseteq \inf _{w \in \mathbb{R}^{n}}\left[t \bar{L}\left(\frac{x-w}{t}\right)+U_{0}(w)\right]=V(t, x)
$$

We prove now that $U(t, x) \subseteq V(t, x)$. The vector-valued function $L$ is $C$-lower and upper continuous, since it is continuous. Let $\eta \in Y(t, x)$. Using Jensen inequality, one has

$$
\bar{L}\left(\frac{1}{t} \int_{0}^{t} \dot{\eta}(s) d s\right) \supseteq \frac{1}{t} \int_{0}^{t} \bar{L}(\dot{\eta}(s)) d s .
$$

Multiplying by $t$, one obtains

$$
t \bar{L}\left(\frac{x-\eta(0)}{t}\right) \supseteq \int_{0}^{t} \bar{L}(\dot{\eta}(s)) d s
$$

and, adding $U_{0}(\eta(0))$,

$$
V(t, x) \supseteq t \bar{L}\left(\frac{x-\eta(0)}{t}\right)+U_{0}(\eta(0)) \supseteq \int_{0}^{t} \bar{L}(\dot{\eta}(s)) d s+U_{0}(\eta(0))=\bar{J}_{t}[\eta] .
$$

Taking the infimum over $\eta \in Y(t, x)$ on the right hand side, we conclude that $V(t, x) \supseteq U(t, x)$.

For any $w \in \mathbb{R}^{n}$ by the domination property there exists a minimizer $w_{0} \in \mathbb{R}^{n}$ such that $\bar{F}_{x, t}\left(w_{0}\right) \supseteq$ $\bar{F}_{x, t}(w)$.

\section{Scalarization}

Throughout this section we suppose that the components of $L$ and $U_{0}$ are such that

$$
\begin{cases}(i) L_{i} \text { is convex and } \lim _{|q| \rightarrow+\infty} L_{i}(q) /|q|=+\infty, & \forall i=1,2, \ldots, d \\ (i i)\left(U_{0}\right)_{i} \text { is Lipschitz on } \mathbb{R}^{n}, & \forall i=1,2, \ldots, d\end{cases}
$$

and that $\bar{F}_{x, t}$ is convex. Here $U$ is $\mathcal{G}\left(\mathbb{R}^{d}, C\right)$ (see Remark 3.3).

Remark 5.1. Under these hypotheses $\inf _{w \in \mathbb{R}^{n}} \bar{F}_{x, t}(w)=\bar{F}_{x, t}\left[\mathbb{R}^{n}\right]$, i.e.

$$
\operatorname{clco} \bigcup_{w \in \mathbb{R}^{n}} \bar{F}_{x, t}(w)=\bigcup_{w \in \mathbb{R}^{n}} \bar{F}_{x, t}(w) .
$$

Let $\zeta \in \operatorname{int} C^{+}$. Then we can consider the infimum of the real-valued function

$$
u_{\zeta}(t, x)=\inf _{w \in \mathbb{R}^{n}} \zeta \cdot\left[t L\left(\frac{x-w}{t}\right)+U_{0}(w)\right] .
$$

Since the components of $\zeta$ are positive,

$$
\lim _{|w| \rightarrow+\infty} \frac{\zeta \cdot L(w)}{|w|}=+\infty
$$

and the infimum is a minimum attained at $w_{\zeta} \in \mathbb{R}^{n}$ and

$$
u_{\zeta}(t, x)=\zeta \cdot\left[t L\left(\frac{x-w_{\zeta}}{t}\right)+U_{0}\left(w_{\zeta}\right)\right]
$$

As an easy result of the classic real-valued methodology (see for example [5]) we have the following proposition and corollary. 
Proposition 5.2. Under the hypotheses (8), for each $\zeta \in \operatorname{int} C^{+}$, the value function in the direction $\zeta u_{\zeta}(x, t)$ is Lipschitz continuous in int $Q_{T}$.

Corollary 5.3. Under the hypotheses (8), for each $\zeta \in \operatorname{int} C^{+}$, the value function in the direction $\zeta$ $u_{\zeta}(x, t)$ is differentiable a.e. in int $Q_{T}$.

The value function in the direction $\zeta u_{\zeta}(t, x)$, if it is differentiable at $(t, x) \in \operatorname{int} Q_{T}$, is a solution of the Hamilton-Jacobi equation:

$$
u_{t}(t, x)+H_{\zeta}(\nabla u(t, x))=0
$$

where

$$
H_{\zeta}(p)=\sup _{w \in \mathbb{R}^{n}}[p \cdot w-\zeta \cdot L(w)]
$$

Proposition 5.4. Let the hypotheses (8) hold, the function $\bar{F}_{x, t}$ be convex and the components of $F_{x, t}(w)$ be strictly convex.

The point $w_{0} \in \mathbb{R}^{n}$ is a minimizer of (7) (in the lattice sense) if and only if there exists $\zeta \in C^{+} \backslash\{0\}$ such that $w_{0}$ is the minimizer of $u_{\zeta}(t, x)$, i.e. $u_{\zeta}(t, x)=\zeta \cdot F_{x, t}\left(w_{0}\right)$.

Proof. If $w_{0}=w_{\zeta}$ for some $\zeta \in \operatorname{int} C^{+}$, let us suppose that there exists $\tilde{w} \in \mathbb{R}^{n}$ such that

$$
\bar{F}_{x, t}(\tilde{w}) \supset \bar{F}_{x, t}\left(w_{\zeta}\right),
$$

then for every $1 \leq i \leq d$ the corresponding components satisfy the inequality

$$
\left(\bar{F}_{x, t}(\tilde{w})\right)_{i} \leq\left(\bar{F}_{x, t}\left(w_{\zeta}\right)\right)_{i}
$$

and at least one inequality is strict. Multiplying each inequality by $\zeta_{i}>0$ and summing up with respect to $i$, we obtain

$$
\zeta \cdot \bar{F}_{x, t}(\tilde{w})<\zeta \cdot \bar{F}_{x, t}\left(w_{\zeta}\right)
$$

which is a contradiction.

Instead, if $w_{0}=w_{\zeta}$ for $\zeta \in C^{+} \backslash \operatorname{int} C^{+}$, then $w_{0}$ is the unique minimizer of a component $\left(F_{x, t}(w)\right)_{i}$ for some $1 \leq i \leq n$. If $\tilde{w} \in \mathbb{R}^{n}$ is such that $\bar{F}_{x, t}(\tilde{w}) \supset \bar{F}_{x, t}\left(w_{0}\right)$, then $\left(F_{x, t}(\tilde{w})\right)_{i} \leq\left(F_{x, t}\left(w_{0}\right)\right)_{i}$ and this means that $w_{0}=\tilde{w}$.

Given $w_{0}$ minimizer of (7), let the point $z_{0} \in \mathbb{R}^{d}$ be the following:

$$
z_{0}=\bar{F}_{x, t}\left(w_{0}\right) .
$$

The point $w_{0}$ is a minimizer of (7) if and only if

$$
\left(z_{0}-C\right) \cap \inf _{w \in \mathbb{R}^{n}} \bar{F}_{x, t}(w)=\left\{z_{0}\right\} .
$$

By Eidelheit's separation theorem, there exists $\zeta \in \mathbb{R}^{d}$ and a real number $s$ such that

$$
\begin{aligned}
\zeta \cdot z & \geq s, \text { for any } z \in \inf _{w \in \mathbb{R}^{n}} \bar{F}_{x, t}(w), \\
\zeta \cdot\left(z_{0}-c\right) & \leq s, \text { for any } c \in C \\
\zeta \cdot\left(z_{0}-c\right) & <s, \text { for any } c \in \operatorname{int} C .
\end{aligned}
$$

It follows that $\zeta \neq 0$. From the first two equations $s=\zeta \cdot z_{0}$. From the last one, we get that $\zeta \cdot z_{0}>\zeta \cdot\left(z_{0}-c\right)$ for any $c \in \operatorname{int} C$. This means that $\zeta \in C^{+} \backslash\{0\}$. 
Proposition 5.5. Let the hypotheses (8) hold, the function $\bar{F}_{x, t}$ be convex and the components of $F_{x, t}(w)$ be strictly convex. Then $\bar{F}_{x, t}\left[\mathbb{R}^{n}\right]$ satisfies the domination property.

Proof. Let $w$ be a point in $\mathbb{R}^{n}$, we want to find a point $w_{0}$ minimizer, such that $\bar{F}_{x, t}\left(w_{0}\right) \supseteq \bar{F}_{x, t}(w)$. Every component $i$ of $F_{x, t}$ admits a minimizer $w_{i} \in \mathbb{R}^{n}$. We consider the vector $z_{0} \in \mathbb{R}^{d}$ whose $i$-th component is the corresponding minimum value $\left(F_{x, t}\left(w_{i}\right)\right)_{i}$. Then $z_{0}+C \supseteq U(t, x)$. Considering the segment between $F_{x, t}(w)$ and $z_{0}$ :

$$
\lambda z_{0}+(1-\lambda) F_{x, t}(w)
$$

for $\lambda \in[0,1]$, there exists

$$
\lambda_{0}=\sup \left\{\lambda \in[0,1] \mid \lambda z_{0}+(1-\lambda) F_{x, t}(w) \in U(x, t)\right\} .
$$

We consider an increasing sequence $\lambda_{m} \rightarrow \lambda_{0}$ and the corresponding vectors $w_{m} \in \mathbb{R}^{n}, c_{m} \in C$ such that

$$
\lambda_{m} z_{0}+\left(1-\lambda_{m}\right) F_{x, t}(w)=F_{x, t}\left(w_{m}\right)+c_{m} .
$$

Since $\lambda_{m} z_{0}+\left(1-\lambda_{m}\right) F_{x, t}(w)$ tends to $\lambda_{0} z_{0}+\left(1-\lambda_{0}\right) F_{x, t}(w)$, the sequences $w_{m}$ and $c_{m}$ must be bounded. It is possible to pass to suitable subsequences (that we still denote $w_{m}$ and $c_{m}$ respectively), that converge. In particular

$$
\lim _{m \rightarrow+\infty} w_{m}=w_{0} \quad \text { and } \quad \lim _{m \rightarrow+\infty} c_{m}=c_{0} .
$$

Taking the limit in both sides of (11), we obtain

$$
\lambda_{0} z_{0}+\left(1-\lambda_{0}\right) F_{x, t}(w)=F_{x, t}\left(w_{0}\right)+c_{0}
$$

and this means that $\lambda_{0} z_{0}+\left(1-\lambda_{0}\right) F_{x, t}(w)$ is in set $U(x, t)$ and that in its boundary $\partial U(x, t)$. Since each component in the curve (10) is decreasing with respect to $\lambda$, we have that

$$
\bar{F}_{x, t}\left(w_{0}\right) \supseteq \bar{F}_{x, t}(w) .
$$

In order to prove that also $F_{x, t}\left(w_{0}\right)$ is in the boundary of $U(x, t)$, given any neighborhood $V$ of $F_{x, t}\left(w_{0}\right), V+c_{0}$ is a neighborhood of $F_{x, t}\left(w_{0}\right)+c_{0}$. There exists $v \in V$, such that $v+c_{0} \notin U(t, x)$, but this implies that also $v \notin U(x, t)$.

Applying the supporting hyperplane theorem to the convex nonempty set $U(x, t)$ at its boundary point $F_{x, t}\left(w_{0}\right)$, one gets $\zeta \neq 0$ such that

$$
\zeta \cdot F_{x, t}\left(w_{0}\right) \leq \zeta \cdot u
$$

for any $u \in U(x, t)$. Since for any $c \in C$, we have

$$
\zeta \cdot F_{x, t}\left(w_{0}\right) \leq \zeta \cdot\left(F_{x, t}\left(w_{0}\right)+c\right),
$$

$\zeta \in C^{+}$.

Remark 5.6. In the hypotheses of the previous proposition, let $w_{0}$ be the minimizer of the $i$-th component of $F_{x, t}$.

Using the classical results, one has that the real fucntion

$$
u_{0}(t, x)=t L_{i}\left(\frac{x-w_{0}}{t}\right)+\left(U_{0}\right)_{i}\left(w_{0}\right)
$$

is a solution of

$$
u_{t}(t, x)+H_{i}(\nabla u(t, x))=0,
$$

where

$$
H_{i}(p)=\sup _{q \in \mathbb{R}^{n}}\left(p \cdot q-L_{i}(q)\right)
$$




\section{Hamilton-Jacobi equation}

For $(t, x) \in Q_{\infty}, q \in \mathbb{R}^{n}$ and $\zeta \in C^{+} \backslash\{0\}$, we write:

$$
\begin{aligned}
U_{t, \zeta}(t, x) & =\lim _{s \rightarrow 0^{+}} \frac{1}{s}\left[U(t+s, x)-{ }_{\zeta} U(t, x)\right], \\
U_{q, \zeta}(t, x) & =\lim _{s \rightarrow 0^{+}} \frac{1}{s}\left[U(t, x+s q)-{ }_{\zeta} U(t, x)\right] .
\end{aligned}
$$

Remark 6.1. From the definition, apart from the extreme cases $\emptyset$ and $\mathbb{R}^{d}$, these derivatives are closed half-spaces with normal $\zeta$.

The definition of the derivative with respect to $x$ in the direction $q$ is stronger than the directional derivative in [12]. More precisely,

$$
U_{q, \zeta}(t, x) \subseteq(U(t, \cdot))_{\zeta}^{\prime}(x, q)
$$

It is also stronger than the definition in [17], where the lower limit of Painlevé-Kuratowski is used.

Proposition 6.2. Let $(t, x) \in Q_{\infty}$. Let $\zeta \in C^{+} \backslash\{0\}$ and let the hypotheses (8) hold. Let the components of $L$ and $U_{0}$ be of class $C^{2}$. Let $L_{\zeta}(w), U_{0, \zeta}(w)$ denote the scalar products

$$
L_{\zeta}(w)=\zeta \cdot L(w), \quad U_{0, \zeta}(w)=\zeta \cdot U_{0}(w),
$$

respectively. If $\hat{w}=\hat{w}(t, x, \zeta) \in \mathbb{R}^{n}$ is such that

$$
\inf _{w \in \mathbb{R}^{n}}\left[t L_{\zeta}\left(\frac{x-w}{t}\right)+U_{0, \zeta}(w)\right]=\left[t L_{\zeta}\left(\frac{x-\hat{w}}{t}\right)+U_{0, \zeta}(\hat{w})\right],
$$

let the following matrix (where $H$ denotes the Hessian matrix) be non-singular

$$
\frac{1}{t} H_{L_{\zeta}}\left(\frac{x-\hat{w}}{t}\right)+H_{U_{0, \zeta}}(\hat{w})
$$

Then the following equations hold:

$$
\begin{aligned}
U_{t, \zeta}(t, x) & =S_{\left(L_{\zeta}\left(\frac{x-\hat{w}}{t}\right)-\nabla L_{\zeta}\left(\frac{x-\hat{w}}{t}\right) \cdot \frac{x-\hat{w}}{t}, \zeta\right)}(1) \\
U_{q, \zeta}(t, x) & =S_{\left(\nabla L_{\zeta}\left(\frac{x-\hat{w}}{t}\right), \zeta\right)}(q) .
\end{aligned}
$$

In particular, if the hypotheses in the previous proposition are realized, the value function admits the derivatives defined in (12) for any $(t, x) \in Q_{\infty}$ and $q \in \mathbb{R}^{n}$.

Proof. If $z \in U_{t, \zeta}(t, x)$, there exists a curve $\left\{z_{s}\right\}_{s \in \mathbb{R}_{+}}$with $z_{s} \in \frac{1}{s}\left[U(t+s, x)-{ }_{\zeta} U(t, x)\right]$ such that $\lim _{s \rightarrow 0^{+}} z_{s}=z$. By definition and by Remark 2.1, $\left\{z_{s}\right\}_{s \in \mathbb{R}_{+}}$is such that

$$
\begin{aligned}
\zeta \cdot z_{s} & +\frac{1}{s} \inf _{w \in \mathbb{R}^{n}}\left[t L_{\zeta}\left(\frac{x-w}{t}\right)+U_{0, \zeta}(w)\right] \\
& \geq \frac{1}{s} \inf _{w \in \mathbb{R}^{n}}\left[(t+s) L_{\zeta}\left(\frac{x-w}{t+s}\right)+U_{0, \zeta}(w)\right] .
\end{aligned}
$$

The minimizer of the right-hand side (it exists by (8)) is a solution of

$$
-\nabla L_{\zeta}\left(\frac{x-w}{t+s}\right)+\nabla U_{0, \zeta}(w)=0
$$


Applying the implicit function theorem to the previous equation, we obtain a $C^{1}$ curve $\hat{w}(s)$, such that

$$
-\nabla L_{\zeta}\left(\frac{x-\hat{w}(s)}{t+s}\right)+\nabla U_{0, \zeta}(\hat{w}(s))=0
$$

and $\hat{w}(0)=\hat{w}$. Using this curve and Taylor's formula we obtain

$$
\begin{aligned}
\zeta \cdot z_{s} & +\frac{1}{s}\left[t L_{\zeta}\left(\frac{x-\hat{w}}{t}\right)+U_{0, \zeta}(\hat{w})\right] \\
\geq & \frac{1}{s}\left[(t+s) L_{\zeta}\left(\frac{x-\hat{w}(s)}{t+s}\right)+U_{0, \zeta}(\hat{w}(s))\right] \\
& =\frac{1}{s}\left[t L_{\zeta}\left(\frac{x-\hat{w}}{t}\right)+U_{0, \zeta}(\hat{w})\right] \\
& +\left[L_{\zeta}\left(\frac{x-\hat{w}}{t}\right)-\nabla L_{\zeta}\left(\frac{x-\hat{w}}{t}\right) \cdot\left(\frac{x-\hat{w}}{t}\right)\right]+o(1) .
\end{aligned}
$$

We conclude that

$$
\zeta \cdot z_{s} \geq\left[L_{\zeta}\left(\frac{x-\hat{w}}{t}\right)-\nabla L_{\zeta}\left(\frac{x-\hat{w}}{t}\right) \cdot \frac{x-\hat{w}}{t}\right]+o(1)
$$

and

$$
z \in\left\{z \in Z \mid \zeta \cdot z \geq\left[L_{\zeta}\left(\frac{x-\hat{w}}{t}\right)-\nabla L_{\zeta}\left(\frac{x-\hat{w}}{t}\right) \cdot\left(\frac{x-\hat{w}}{t}\right)\right]\right\} .
$$

Vice versa, if $z$ is such that

$$
\zeta \cdot z \geq\left[L_{\zeta}\left(\frac{x-\hat{w}}{t}\right)-\nabla L_{\zeta}\left(\frac{x-\hat{w}}{t}\right) \cdot\left(\frac{x-\hat{w}}{t}\right)\right]=\mu_{t, x, \zeta}
$$

it can be written as $z=\mu \zeta+z_{1}$, where $\mu \geq \mu_{t, x, \zeta}$ and $z_{1}$ is perpendicular to $\zeta$. We consider the following curve

$$
\begin{aligned}
z_{s}= & \frac{1}{s}\left[\inf _{w \in \mathbb{R}^{n}}\left((t+s) L_{\zeta}\left(\frac{x-w}{t+s}\right)+U_{0, \zeta}(w)\right)\right. \\
& \left.-\inf _{w \in \mathbb{R}^{n}}\left(t L_{\zeta}\left(\frac{x-w}{t}\right)+U_{0, \zeta}(w)\right)\right] \zeta+\left(\mu-\mu_{t, x, \zeta}\right) \zeta+z_{1}
\end{aligned}
$$

that converges to $z \in U_{t, \zeta}(t, x)$.

For equation (16), if $\tilde{z} \in U_{q, \zeta}(t, x)$, there exists a curve $\left\{\tilde{z}_{s}\right\}_{s \in \mathbb{R}_{+}}$with $\tilde{z}_{s}$ converging to $\tilde{z}$ such that $\tilde{z}_{s} \in \frac{1}{s}\left[U(t, x+s q)-{ }_{\zeta} U(t, x)\right]$. We have that

$$
\begin{aligned}
\zeta \cdot \tilde{z}_{s} & +\frac{1}{s} \inf _{w \in \mathbb{R}^{n}}\left[t L_{\zeta}\left(\frac{x-w}{t}\right)+U_{0, \zeta}(w)\right] \\
& \geq \frac{1}{s} \inf _{w \in \mathbb{R}^{n}}\left[t L_{\zeta}\left(\frac{x+s q-w}{t}\right)+U_{0, \zeta}(w)\right] .
\end{aligned}
$$

The minimizer of the right-hand side is a solution of

$$
-\nabla L_{\zeta}\left(\frac{x+s q-w}{t}\right)+\nabla U_{0, \zeta}(w)=0 .
$$


Applying the implicit function theorem to the previous equation, we obtain a $C^{1}$ curve $\tilde{w}(s)$, such that $\tilde{w}(0)=\hat{w}$. As before, we obtain

$$
\begin{aligned}
\zeta \cdot \tilde{z}_{s} & +\frac{1}{s}\left[t L_{\zeta}\left(\frac{x-\hat{w}}{t}\right)+U_{0, \zeta}(\hat{w})\right] \\
& \geq \frac{1}{s}\left[t L_{\zeta}\left(\frac{x+s q-\tilde{w}(s)}{t}\right)+U_{0, \zeta}(\tilde{w}(s))\right] \\
& =\frac{1}{s}\left[t L_{\zeta}\left(\frac{x-\hat{w}}{t}\right)+U_{0, \zeta}(\hat{w})\right]+\nabla L_{\zeta}\left(\frac{x-\hat{w}}{t}\right) \cdot q+o(1) .
\end{aligned}
$$

We may conclude that

$$
\zeta \cdot \tilde{z}_{s} \geq \nabla L_{\zeta}\left(\frac{x-\hat{w}}{t}\right) \cdot q+o(1)
$$

and

$$
\tilde{z} \in\left\{z \in Z \mid \zeta \cdot z \geq \nabla L_{\zeta}\left(\frac{x-\hat{w}}{t}\right) \cdot q\right\} .
$$

The other inclusion is similar to the previous case.

Remark 6.3. (i) We have that

$$
\lim _{s \rightarrow 0^{+}} \frac{1}{s}\left[U(t+s, x+s q)-{ }_{\zeta} U(t+s, x)\right]=U_{q, \zeta}(t, x) .
$$

(ii) It is easy to see that

$$
\begin{aligned}
\left(U(t+s, x+s q)-{ }_{\zeta} U(t+s, x)\right) & +\left(U(t+s, x)-{ }_{\zeta} U(t, x)\right) \\
& =U(t+s, x+s q)-{ }_{\zeta} U(t, x) .
\end{aligned}
$$

From (16) and (1) we deduce that

$$
U_{q, \zeta}(t, x)=\left(\nabla L_{\zeta}\left(\frac{x-\hat{w}}{t}\right) \cdot q\right) \hat{z}+H^{+}(\zeta),
$$

for $\hat{z} \in \mathbb{R}^{d}$ such that $\zeta \cdot \hat{z}=1$, that is the set $U_{q, \zeta}(t, x)$ is described by a vector: then we write

$$
U_{q, \zeta}(t, x)=\left(\nabla U_{\zeta}(x, t) \cdot q\right) \hat{z}+H^{+}(\zeta),
$$

where

$$
\nabla U_{\zeta}(x, t)=\nabla L_{\zeta}\left(\frac{x-\hat{w}}{t}\right)
$$

Theorem 6.4. Let $(t, x) \in Q_{\infty}$. Let $\zeta \in C^{+} \backslash\{0\}$ and let the hypotheses (8) hold. Let the components of $L$ and $U_{0}$ be of class $C^{2}$. If $\hat{w} \in \mathbb{R}^{n}$ is as in (13), let the matrix (14) be non-singular.

Then $U(t, x)$ is a solution of

$$
U_{t, \zeta}(t, x)+\mathcal{H}\left(\nabla U_{\zeta}(t, x), \zeta\right)=H^{+}(\zeta)
$$

where

$$
\mathcal{H}(p, \zeta)=\bar{L}^{*}(p, \zeta)
$$

for any $p \in \mathbb{R}^{n}, \zeta \in C^{+} \backslash\{0\}$. Moreover, $U(t, x)$ is a solution of

$$
\sup _{\zeta \in C^{+} \backslash\{0\}}\left[U_{t, \zeta}(t, x)+\mathcal{H}\left(\nabla U_{\zeta}(t, x), \zeta\right)\right]=C .
$$


Equations (17) and (18) are the Hamilton-Jacobi equations of the given problem.

Proof. Given $q \in \mathbb{R}^{n}, s>0$, we set $y(\tau)=x+(\tau-t) q$. From (5), we obtain

$$
U(t+s, x+s q) \supseteq \int_{t}^{t+s} \bar{L}(\dot{y}(\tau)) d \tau+U(t, x),
$$

and consequently

$$
\frac{1}{s}\left(U(t+s, x+s q)-{ }_{\zeta} U(t, x)\right) \supseteq \bar{L}(q)+H^{+}(\zeta) .
$$

In the limit, we conclude that for any $q \in \mathbb{R}^{n}$

$$
U_{t, \zeta}(t, x)+U_{q, \zeta} \supseteq \bar{L}(q)+H^{+}(\zeta) .
$$

From (19) and Lemma 2.2 we can deduce that for any $q \in \mathbb{R}^{n}$

$$
U_{t, \zeta}(t, x)+\left(U_{q, \zeta}-{ }_{\zeta} \bar{L}(q)\right) \supseteq H^{+}(\zeta) .
$$

To prove that

$$
U_{t, \zeta}(t, x)+\bigcap_{q \in \mathbb{R}^{n}}\left(U_{q, \zeta}-\zeta \bar{L}(q)\right) \supseteq H^{+}(\zeta),
$$

let us consider $h \in H^{+}(\zeta)$ such that, for any $z \in U_{t, \zeta}(t, x)$, there holds $h-z \notin \bigcap_{q \in \mathbb{R}^{n}}\left(U_{q, \zeta}-\zeta \bar{L}(q)\right)$. In particular, for $z$ that minimizes $\inf \zeta \cdot U_{t, \zeta}(t, x)$, there exists $q_{0} \in \mathbb{R}^{n}$ and $h-z \notin U_{q_{0}, \zeta}-{ }_{\zeta} \bar{L}\left(q_{0}\right)$. Then $\zeta \cdot(h-z)+L_{\zeta}\left(q_{0}\right)<\inf \zeta \cdot U_{q_{0}, \zeta}$ and

$$
\begin{aligned}
\zeta \cdot h & <\inf \zeta \cdot U_{t, \zeta}(t, x)+\inf \zeta \cdot U_{q_{0}, \zeta}-L_{\zeta}\left(q_{0}\right) \\
& =L_{\zeta}\left(\frac{x-\hat{w}}{t}\right)+\nabla L_{\zeta}\left(\frac{x-\hat{w}}{t}\right) \cdot\left(q_{0}-\frac{x-\hat{w}}{t}\right)-L_{\zeta}\left(q_{0}\right) \leq 0 .
\end{aligned}
$$

This is a contradiction to $h$ being in $H^{+}(\zeta)$ and proves (20).

From (15) and (16) we can see that

$$
U_{t, \zeta}(t, x)+U_{\frac{x-\hat{w}}{t}, \zeta}=\bar{L}\left(\frac{x-\hat{w}}{t}\right)+H^{+}(\zeta) .
$$

In order to prove the opposite inclusion in (20), we consider that

$$
U_{t, \zeta}(t, x)+\bigcap_{q \in \mathbb{R}^{n}}\left(U_{q, \zeta}-\zeta \bar{L}(q)\right) \subseteq U_{t, \zeta}(t, x)+\left(U_{\frac{x-\hat{w}}{t}, \zeta}-{ }_{\zeta} \bar{L}\left(\frac{x-\hat{w}}{t}\right)\right) .
$$

Now, by the second implication in Lemma 2.2 and (21), we conclude that

$$
U_{t, \zeta}(t, x)+\left(U_{\frac{x-\hat{w}}{t}, \zeta}-\zeta \bar{L}\left(\frac{x-\hat{w}}{t}\right)\right) \subseteq H^{+}(\zeta) .
$$

We have proved that

$$
U_{t, \zeta}(t, x)+\bigcap_{q \in \mathbb{R}^{n}}\left(U_{q, \zeta}-\zeta \bar{L}(q)\right)=H^{+}(\zeta) .
$$



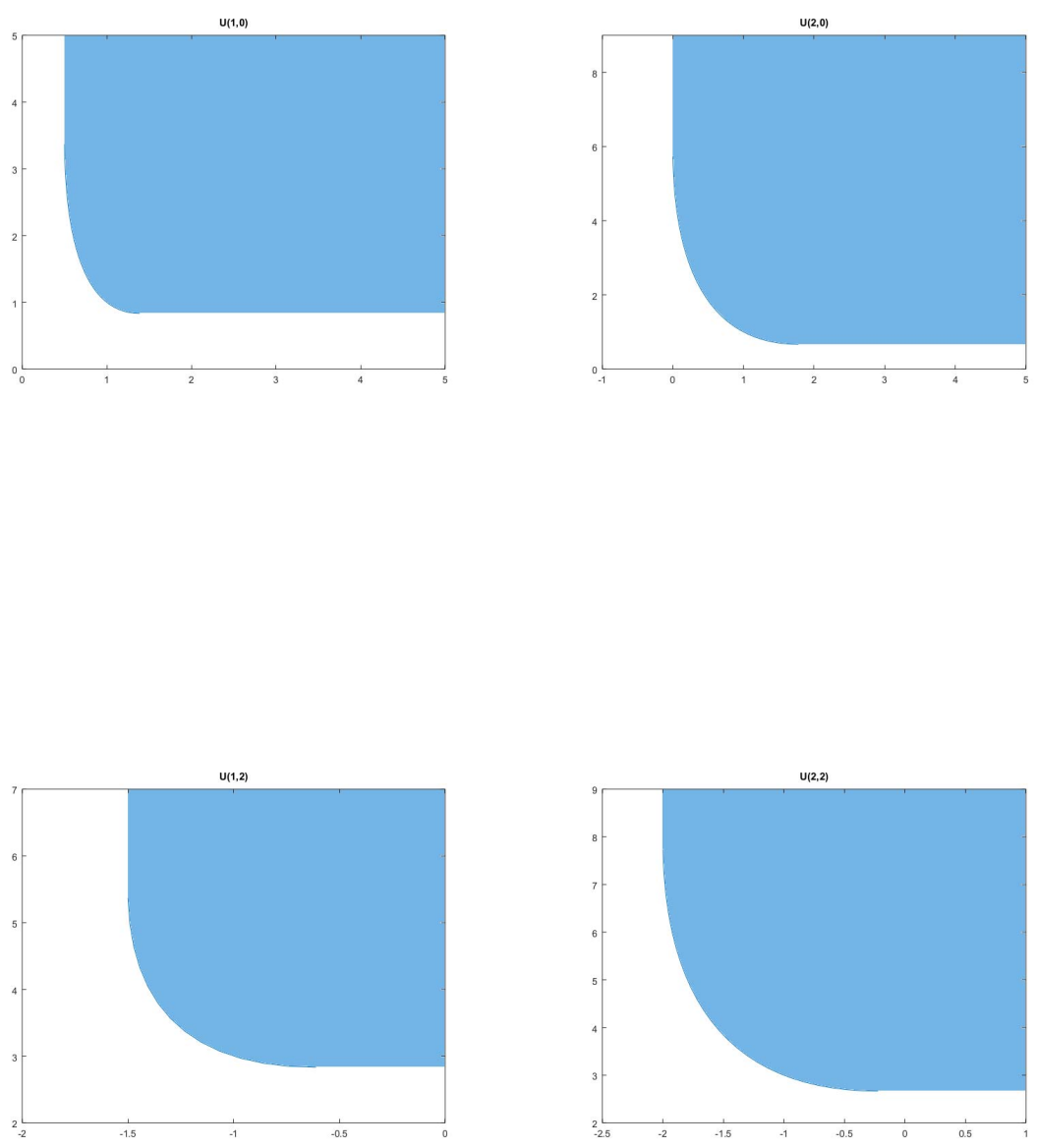

Figure 1: The four sets are respectively $U(1,0), U(2,0), U(1,2)$ and $U(2,2)$. 
Example 6.5. Let us consider $L, U_{0}: \mathbb{R} \rightarrow \mathbb{R}^{2}$ defined by

$$
L(w)=\left(\begin{array}{c}
\frac{1}{2} w^{2} \\
\frac{3}{2} w^{2}
\end{array}\right) \quad \text { and } \quad U_{0}=\left(\begin{array}{c}
1-w \\
1+w
\end{array}\right)
$$

and $C=\mathbb{R}_{+}^{2}$. Some images of the value function are in Figure 1.

Given $\zeta=\left(\zeta_{1}, \zeta_{2}\right) \in C^{+} \backslash\{0\}$, then $\hat{w}=\hat{w}(t, x, \zeta)$ defined in (13) is

$$
\hat{w}=x+t \frac{\zeta_{1}-\zeta_{2}}{\zeta_{1}+3 \zeta_{2}}
$$

Hypothesis (14) is fulfilled, in fact:

$$
\frac{1}{t} H_{L_{\zeta}}\left(\frac{x-\hat{w}}{t}\right)+H_{U_{0, \zeta}}(\hat{w})=\frac{1}{t}\left(\zeta_{1}+3 \zeta_{2}\right)>0 .
$$

Then it is possible to compute (15) and (16):

$$
\begin{aligned}
& U_{t, \zeta}(t, x)=\left\{z \in Z \mid \zeta \cdot z \geq-\frac{1}{2} \frac{\left(\zeta_{1}-\zeta_{2}\right)^{2}}{\zeta_{1}+3 \zeta_{2}}\right\} \\
& U_{q, \zeta}(t, x)=\left\{z \in Z \mid \zeta \cdot z \geq-\left(\zeta_{1}-\zeta_{2}\right) q\right\} .
\end{aligned}
$$

For example for $(t, x)=(1,0)$ and some values of $\zeta$ we obtain the following sets:

$$
\begin{aligned}
& \zeta=(1,0) \quad \begin{array}{l}
U_{t, \zeta}(1,0)=\left\{\left(z_{1}, z_{2}\right) \mid z_{1} \geq-\frac{1}{2}\right\} \\
U_{q, \zeta}(1,0)=\left\{\left(z_{1}, z_{2}\right) \mid z_{1} \geq-q\right\}
\end{array} \\
& \zeta=(1,1) \quad \begin{array}{l}
U_{t, \zeta}(1,0)=\left\{\left(z_{1}, z_{2}\right) \mid z_{1}+z_{2} \geq 0\right\} \\
U_{q, \zeta}(1,0)=\left\{\left(z_{1}, z_{2}\right) \mid z_{1}+z_{2} \geq 0\right\}
\end{array} \\
& \zeta=(0,1) \quad \begin{array}{l}
U_{t, \zeta}(1,0)=\left\{\left(z_{1}, z_{2}\right) \mid z_{2} \geq-\frac{1}{6}\right\} \\
U_{q, \zeta}(1,0)=\left\{\left(z_{1}, z_{2}\right) \mid z_{2} \geq q\right\}
\end{array}
\end{aligned}
$$

The Hamiltonian function is:

$$
\begin{aligned}
\mathcal{H}_{\zeta}(p) & =\bigcap_{q \in \mathbb{R}}\left(S_{(p, \zeta)}(q)-{ }_{\zeta} \bar{L}(q)\right) \\
& =\left\{z \in Z \mid \zeta \cdot z \geq \sup _{q \in \mathbb{R}}\left[p q-L_{\zeta}(q)\right]\right\} \\
& =\left\{z \in Z \mid \zeta \cdot z \geq \frac{p^{2}}{2\left(\zeta_{1}+3 \zeta_{2}\right)}\right\} .
\end{aligned}
$$

\section{Real-valued case}

In the particular case $d=1$, so when the objective function is real-valued, the value function can be written as

$$
U(t, x)=u(t, x)+\mathbb{R}_{+},
$$

where $u(t, x)$ is the classical value function. If the hypotheses hold and $u(t, x)$ is differentiable, it is easy to see that

$$
\begin{aligned}
U_{t, 1}(t, x) & =u_{t}(t, x)+\mathbb{R}_{+}, \\
U_{q, 1}(t, x) & =\nabla u(t, x) \cdot q+\mathbb{R}_{+} .
\end{aligned}
$$


Hypothesis (14) becomes

$$
\frac{1}{t} L^{\prime \prime}\left(\frac{x-\hat{w}}{t}\right)+U_{0}^{\prime \prime}(\hat{w})>0,
$$

since $\hat{w}$ is a minimizer.

Since

$$
S_{(p, 1)}(q)=p \cdot q+\mathbb{R}_{+},
$$

the Hamiltonian function is

$$
\mathcal{H}_{1}(p)=\sup _{q \in \mathbb{R}^{n}}(p \cdot q-L(q))+\mathbb{R}_{+}
$$

and equations (17) and (18) both become

$$
u_{t}(t, x)+\mathcal{H}_{1}\left(\nabla L\left(\frac{x-\hat{w}}{t}\right)\right)=\mathbb{R}_{+} .
$$

Using (16) and the second equation in (22), the previous equation can be written

$$
u_{t}(t, x)+\mathcal{H}_{1}(\nabla u(t, x))=\mathbb{R}_{+}
$$

and the real-valued case is recovered.

\section{References}

[1] J.P. Aubin, H. Frankowska, Set-Valued Analysis, Birkhäuser, Boston-Basel-Berlin 1990.

[2] R.J. Aumann, Integrals of set-valued functions, J. Math. Anal. Appl. 12 (1965), 1-12.

[3] E.N. Barron, R. Jensen, W. Liu, Hopf-Lax-type formula for $u_{t}+H(u, D u)=0$, Journal of Differential Equations 1261 (1996), 48-61.

[4] H. Bonnel, C.Y. Kaya, Optimization over the efficient set of multi-objective convex optimal control problems, Journal of Optimization Theory and Applications 1471 (2010), 93-112.

[5] P. Cannarsa, C. Sinestrari, Semiconcave Functions, Hamilton-Jacobi Equations, and Optimal Control, Progress in Nonlinear Differential Equations and Their Applications, Birkhäuser (2004).

[6] N. Caroff, Multicriteria Optimal Control and Vectorial Hamilton-Jacobi Equation, in: LargeScale Scientific Computing, LSSC 2007, Eds. I. Lirkov, S. Margenov and J. Waśniewski, Lecture Notes in Computer Science 4818, Springer, Berlin, Heidelberg, 293-299.

[7] M. Ehrgott, Multicriteria Optimization, 2nd edition, Springer Science \& Business Media (2005).

[8] L.C. Evans, Partial Differential Equations, Graduate Studies in Mathematics 19, Providence, RI, American Mathematical Society (2010).

[9] A. Göpfert, H. Riahi, C. Tammer, C. Zalinescu, Variational Methods in Partially Ordered Spaces, Springer Science \& Business Media (2003).

[10] A. Guigue, Set-valued return function and generalized solutions for multiobjective optimal control problems (MOC), SIAM Journal on Control and Optimization 513 (2013), 2379-2405. 
[11] A.H. Hamel, F. Heyde, A. Löhne, B. Rudloff, C. Schrage (Eds.), Set Optimization and Applications - The State of the Art, Springer Proceedings in Mathematics and Statistics 151 (2015).

[12] A.H. Hamel, C. Schrage, Directional derivatives, subdifferentials and optimality conditions for set-valued convex functions, Pacific Journal of Optimization 104 (2014), 667-689.

[13] N. Katzourakis, T. PrYer, On Hamilton-Jacobi-Bellman equations arising in deterministic and stochastic optimal control with vectorial cost, arXiv:1409.8648 (2014), 1-18.

[14] P.L. Lions, Generalized Solutions of Hamilton-Jacobi Equations, Research Notes In mathematics Series 69, Pitman Publishing (1982).

[15] J. Matkowski, K. Nikodem, An integral Jensen inequality for convex multifunctions, Results in Mathematics 26 3-4 (1994), 348-353.

[16] W. MARKS, Multicriteria optimisation of shape of energy-saving buildings, Building and Environment 324 (1997), 331-339.

[17] M. PILECKA, Set-valued optimization and its application to bilevel optimization, PhD-thesis 2016, Technische Universität Bergakademie Freiberg.

[18] R.W. ReID, S.J. Citron, On noninferior performance index vectors, Journal of Optimization Theory and Applications 71 (1971), 11-28.

[19] R.B. Vinter, Optimal Control, Boston, Publisher: Birkhauser (2000).

[20] J.J. Wang, Y.Y. Jing, C.F. Zhang, J.H. ZhaO, Review on multi-criteria decision analysis aid in sustainable energy decision-making, Renewable and Sustainable Energy Reviews 139 (2009), 2263-2278.

[21] P.L. Yu, G. Leitmann, Nondominated decisions and cone convexity in dynamic multicriteria decision problems, Journal of Optimization Theory and Applications 145 (1974), 573-584.

[22] P.L. Yu, G. Leitmann, Compromise solutions, domination structures, and Salukvadze's solution, Journal of Optimization Theory and Applications 133 (1974), 362-378.

[23] L. ZADEH, Optimality and non-scalar-valued performance criteria, IEEE Transactions on Automatic Control 81 (1963), 59-60. 